\section{Análise de sobrevida global em pacientes diagnosticados com carcinoma de células escamosas de boca no INCA no ano de 1999}

\section{Overall survival analysis in oral squamous cell carcinoma patients diagnosed at the National Cancer Institute in 1999}

Julia Honorato ${ }^{1}$

Danielle Resende Camisasca'

Licínio Esmeraldo da Silva ${ }^{2}$

Fernando Luiz Dias ${ }^{3}$

Paulo Antônio Silvestre de Faria ${ }^{4}$

Simone de Queiroz Chaves Lourenço'

${ }^{1}$ Programa de Pós-Graduação em Patologia - Universidade Federal Fluminense (UFF)

${ }^{2}$ Departamento de Estatística da Universidade Federal Fluminense.

${ }^{3}$ Serviço de Cirurgia de Cabeça e Pescoço do Instituto Nacional de Câncer

${ }^{4}$ Divisão de Patologia do Instituto Nacional de Câncer

O presente trabalho foi realizado com o apoio do Conselho Nacional de Desenvolvimento Científico e Tecnológico - CNPq - Brasil.

Correspondência: Júlia Honorato. R. Coronel Moreira Cesar 322/406 - Icaraí - Niterói - RJ CEP: 24230-064.E-mail: julia_honorato@yahoo.com.br

\section{Resumo}

O carcinoma de células escamosas de boca compreende cerca de 90 a $95 \%$ de todas as neoplasias malignas da boca e é um dos tipos de câncer mais frequentes no Brasil. O índice de sobrevida em 5 anos é baixo e permaneceu estável nas últimas décadas, apesar dos avanços nas terapias. O objetivo deste estudo foi analisar o perfil e a sobrevida global dos pacientes diagnosticados com carcinoma de células escamosas de boca no ano de 1999 no Instituto Nacional de Câncer. Dos 320 pacientes incluídos no estudo, 79,4\% eram homens. A idade média foi de 56,7 anos, e $82,2 \%$ deles fumavam e/ ou bebiam. A língua, seguida do assoalho de boca foram os locais mais acometidos. A maioria $(68,9 \%)$ dos pacientes foi diagnosticada em estádios tardios e submetida à radioterapia exclusiva (53,6\%). A sobrevida média no período do estudo foi de 29,4 meses. Os pacientes dos estádios iniciais apresentaram maior sobrevida, assim como aqueles submetidos apenas à cirurgia como forma de tratamento e os que não apresentaram linfonodos acometidos ao diagnóstico. Tumores localizados em palato duro e mucosa jugal apresentaram pior prognóstico. Foram fatores preditivos independentes de melhor sobrevida os tumores T1 ou T2 ( $\mathrm{p}=0,001)$, sem acometimento de linfonodos ( $\mathrm{p}=0,012)$ e não localizados em mucosa jugal $(\mathrm{p}=0,021)$. O diagnóstico do câncer oral ainda se faz em estádios tardios, o que influencia negativamente a sobrevida global dos pacientes. Maior ênfase deve ser dada à capacitação dos profissionais para o reconhecimento precoce do câncer e à conscientização da população de risco.

Palavras- chave: Câncer da boca. Carcinoma de células escamosas. Análise de sobrevida. Prognóstico. 


\section{Abstract}

Oral squamous cell carcinoma accounts for 90 to $95 \%$ of all malignant neoplasms of the mouth and it is one of most common cancers in Brazil. Five-year survival rates remain low and have not improved over the past decades, despite advances in therapy. The purpose of this study was to analyze the features and overall survival of patients diagnosed with oral squamous cell carcinoma in 1999 at the National Institute of Cancer. Male patients (79.4\%) with a medium age of 56.7 years, with smoking or drinking habits (82.2\%) comprised most of the sample. Tongue and floor of the mouth were the most prevalent sites of occurrence. Patients were diagnosed mainly at advanced stages of the disease $(68.9 \%$ stages III and IV) and were treated with radiation therapy alone (53.6\%). Mean survival was 29.4 months, and patients diagnosed in early stages had better survival rates, as well as those who were surgically treated and those who did not show cervical node metastasis at diagnosis. Tumors located in the palate or in the buccal mucosa presented the worst prognosis. Cox regression analysis showed that $\mathrm{T} 1$ or $\mathrm{T} 2$ lesions $(\mathrm{p}=0.001$ ), with no neck metastasis $(\mathrm{p}=0.012)$ and anatomic site other than buccal mucosa $(\mathrm{p}=0.021)$ were independent predictive factors of a better survival rate. Oral cancer diagnosis still occurs in advanced stages, and it negatively influences overall survival, so there should be efforts to prepare and train professionals to recognize lesions at early stages and to make the population at greatest risk aware of the disease.

Keywords: Squamous cell carcinoma. Oral cancer. Prognosis. Survival analysis.

\section{Introdução}

A mortalidade por doenças crônico-degenerativas vem mostrando uma ascensão progressiva, destacando-se, entre elas, as neoplasias malignas, que correspondem à segunda causa de morte no Brasil, excluindo as causas externas. O câncer de boca está entre os dez tipos de câncer mais frequentes no Brasil e a estimativa de incidência para 2009 no Brasil aponta esse tumor como o $5^{\circ}$ mais frequente entre os homens (com 10.380 casos estimados) e o $7^{\circ}$ entre as mulheres (com 3.780 casos estimados) ${ }^{1}$.

A partir desses dados, constata-se que o câncer de boca se mostra como um problema de saúde pública e a realização do diagnóstico precoce e do pronto tratamento representam não só uma forma de prevenção secundária, mas também um meio de aumentar a sobrevida dos pacientes acometidos.

Para o carcinoma de células escamosas (CCE) de boca, o estadiamento clínico e a localização do tumor, assim como a gradação histopatológica de malignidade demonstram ser importantes indicadores de prognóstico ${ }^{2,3}$. A localização anatômica da lesão deve ser considerada como um bom indicador, já que os tumores apresentam comportamentos diferentes dependendo da sua localização².

O tratamento dos tumores depende, de modo geral, da localização, do estadiamento clínico, da gradação histopatológica e das condições físicas do paciente, sendo realizado através de três modalidades: cirurgia, radioterapia e quimioterapia, de maneira individualizada ou em associação.

O índice de sobrevida em 5 anos é baixo e não apresentou grandes alterações nas últimas três décadas, apesar dos avanços na terapia ${ }^{4}$. O câncer de boca tem uma taxa de $50 \%$ de sobrevida em 5 anos, ou menos. Esta taxa pode ser aumentada quando as lesões são diagnosticadas em estádios menos avançados. Por isso, acredita-se que detectar o câncer de boca em estágio precoce é o meio mais efetivo de reduzir a morbidade, complexidade e magnitude do tratamento ${ }^{5-7}$. 
Os estudos descritos indicam o sexo masculino como o grupo mais afetado pelo carcinoma de células escamosas em boca, assim como a faixa etária de 50 a 60 anos e a língua como a localização anatômica mais frequente. A análise da sobrevida não é comumente realizada. Além disso, há informações divergentes quanto às múltiplas formas de avaliação de prognóstico. $\mathrm{O}$ presente estudo teve como objetivo geral analisar a possível associação dos fatores de risco e prognósticos com a sobrevida do carcinoma de células escamosas (CCE) de boca.

\section{Material e métodos}

\section{Pacientes}

O grupo analisado constituiu-se de 320 indivíduos e foi obtido a partir de prontuários de pacientes diagnosticados com carcinoma de células escamosas (CCE) de boca no período de $1^{\circ}$ de janeiro a 31 de dezembro de 1999, do Instituto Nacional do Câncer (INCA), Rio de Janeiro - RJ. Este trabalho foi aprovado pelo Comitê de Ética e Pesquisa com número de registro CEP no 038/06.

De todos os pacientes cadastrados no banco de dados da instituição, durante o período de estudo, 616 foram diagnosticados através do exame histopatológico ou citopatológico com carcinoma de células escamosas de boca e orofaringe. Inicialmente, foram excluídos 49 pacientes com CCE de lábio e 90 com CCE de orofaringe, e 3 pacientes com carcinoma verrrucoso. Os 474 prontuários restantes foram solicitados para análise mais detalhada, e para isso foram adotados os seguintes critérios de exclusão:

- Carcinomas de células escamosas tendo como sítio inicial orofaringe e lábio inferior, devido à comprovada etiologia diferenciada dos tumores de lábio e por não ser a orofaringe considerada nos limites da cavidade oral;

- Pacientes não tratados na instituição devido a pequenas variações nos protocolos usados nas diversas instituições para tratamento de um mesmo tipo de lesão;

- Toda a amostra teve o diagnóstico confirmado por análise histopatológica, seja por biópsia realizada na instituição ou através de revisão de lâmina encaminhada de outros centros.

Em cada um dos 320 prontuários adequados à metodologia deste trabalho, foram avaliados os dados sócio-demográficos, comportamentais, clínico-patológicos e de sobrevida das variáveis descritas a seguir:

\section{Dados sócio-demográficose comportamentais}

Foram obtidos dos prontuários os seguintes dados sócio-demográficos: sexo, idade e raça; além destes, os dados relacionados aos hábitos comportamentais: tabagismo e etilismo.

\section{Dados clínico-patológicos}

Foram avaliados os dados de estadiamento clínico, localização do tumor, tratamento, intervalo entre diagnóstico e tratamento e gradação histopatológica.

O Estadiamento Clínico da doença (Classificação TNM) foi baseado na descrição clínica da lesão, metástases regionais em linfonodos $(\mathrm{N})$ e metástases à distância (M), conforme arquivado nos prontuários dos pacientes. O estadiamento TNM inicial do paciente considerado foi o descrito no prontuário, geralmente definido na primeira consulta. A classificação TNM clínica foi agrupada em estádios de I a IV, conforme a $6^{a}$ edição da União Internacional de Combate ao Câncer (UICC). Os linfonodos regionais acometidos foram descritos da seguinte forma: Nx para linfonodos regionais que não podem ser avaliados; N0, na ausência de metástase em linfonodos regionais; e N1, N2 e N3 onde houve comprometimento crescente dos linfonodos. Para fins de agrupamento por estádios, os pacientes classificados como Mx foram considerados como M0, já que os pacientes, em consultas posteriores, passam por diversos exames, 
incluindo radiografia de tórax, para verificar a presença de metástases. Todos os pacientes foram tratados na mesma instituição, seguindo a mesma filosofia ${ }^{8}$.

\section{Dados de sobrevida}

A sobrevida global foi calculada a partir da data do diagnóstico histopatológico. A informação de sobrevida foi obtida de acordo com as consultas de acompanhamento clínico registradas nos prontuários, até a data da última consulta, quer o paciente estivesse livre de doença ou não, ou até a data de óbito registrada no prontuário. $\mathrm{O}$ mês de setembro de 2006 foi fixado como limite para o acompanhamento dos registros de sobrevida nos prontuários.

\section{Análise Estatística}

As descrições estatísticas das características colhidas dos prontuários foram apresentadas através de proporções ou médias ( \pm erro padrão), conforme a natureza das variáveis.

O método Kaplan-Meier foi utilizado para a análise de sobrevida e o teste log rank para comparação das curvas. A análise de regressão de Cox avaliou as variáveis significativas pelo método de Kaplan-Meier para determinar o valor independente de cada uma em relação à sobrevida.

A associação de variáveis sócio-demográficas e clínico-patológicas com o estádio clínico, com a gradação histopalógica da OMS e com o óbito, foi analisada pelo teste do qui-quadrado ou pelo teste exato de Fisher, quando aplicável.

As decisões estatísticas foram tomadas ao nível de significância de 0,05 e para a análise estatística foi utilizado o software SPSS (Statistic Package for Social Sciences) versão 10.0 .

\section{Resultados}

Do total de 320 pacientes incluídos no estudo, 254 eram homens $(79,4 \%)$ e 66 eram mulheres $(20,6 \%)$. A idade média do grupo analisado foi de 56,7 anos, com erro padrão de 12,9 e mediana de 57 anos. Os pacientes da raça branca foram os mais acometidos (47\%), seguidos dos pardos $(37,5 \%)$ e dos negros (15,5\%). As características do grupo estudado estão apresentadas na Tabela 1.

O número de tumores foi 520, excedendo o número de pacientes devido à possibilidade de o tumor acometer mais de um sítio anatômico. Quanto à localização, o sítio anatômico mais acometido foi a língua. Dos 320 pacientes com CCE de boca, 78,7\% (n = 252) foram tratados na instituição, 20,3\% $(n=65)$ não foram submetidos às formas tradicionais de terapia, e em $0,9 \%(\mathrm{n}=3)$ dos casos não foi encontrado nenhum registro sobre a forma de tratamento. Dentre os 252 pacientes tratados, $53,6 \%(n=135)$ foram submetidos exclusivamente a radioterapia e $12,7 \%(\mathrm{n}=32$ ) foram submetidos a cirurgia isoladamente. As associações entre tratamentos, como cirurgia associada à radioterapia ou radioterapia associada à quimioterapia, totalizaram $33,7 \%(n=85)$ do total de casos. Dos 65 pacientes que foram admitidos neste centro de referência, mas não receberam as terapias convencionais de tratamento, 56,9\% ( $n=37)$ abandonaram o tratamento após o diagnóstico da doença, $38,4 \%(\mathrm{n}=25)$ foram submetidos a cuidados paliativos e $4,7 \%(\mathrm{n}=3)$ morreram antes do início do tratamento.

O tempo médio de intervalo entre o diagnóstico e o início do tratamento na instituição foi de 2,6 meses, sendo o tempo máximo de espera de 22,6 meses e o tempo mínimo de 0 meses, correspondendo aos pacientes que foram submetidos a biópsia por congelação e operados no mesmo tempo cirúrgico.

A Tabela 2 resume os resultados da análise da associação das variáveis: sexo, idade, raça, tabagismo, etilismo, localização, tratamento e tempo de espera, que foram correlacionadas com as variáveis: estádio clínico, gradação histopatológica da OMS e óbito.

O estadiamento clínico demonstrou associação significativa com a raça $(\mathrm{p}=0,025)$, com a localização em mucosa jugal $(\mathrm{p}=0,029)$, gengiva inferior $(\mathrm{p}=0,015) \mathrm{e}$ 
Tabela 1 - Características clínico-patológicas da amostra ( $\mathrm{n}=320)$

Table 1 - Clinical-pathological data of the sample $(n=320)$

\begin{tabular}{|c|c|c|}
\hline VARIÁVEL & $\mathrm{n}$ & $\%$ \\
\hline \multicolumn{3}{|l|}{ Idade } \\
\hline$<60$ & 183 & 57,18 \\
\hline$\geq 60$ & 125 & 39,06 \\
\hline Sem informação & 12 & 3,76 \\
\hline \multicolumn{3}{|l|}{ Sexo } \\
\hline Feminino & 66 & 20,62 \\
\hline Masculino & 254 & 79,38 \\
\hline \multicolumn{3}{|l|}{ Raça } \\
\hline Branco & 149 & 46,57 \\
\hline Pardo & 119 & 37,2 \\
\hline Negro & 49 & 15,3 \\
\hline Sem informação & 3 & 0,93 \\
\hline \multicolumn{3}{|l|}{ Tabagismo e Etilismo } \\
\hline Tabagista & 5 & 1,56 \\
\hline Etilismo & 58 & 18,12 \\
\hline Tabagismo e Etilismo & 204 & 63,75 \\
\hline Tabagismo ou Etilismo & 53 & 16,56 \\
\hline Sem informação & 3 & 0,01 \\
\hline \multicolumn{3}{|l|}{ Estádio Clínico } \\
\hline I & 22 & 6,88 \\
\hline II & 58 & 18,12 \\
\hline III & 57 & 17,82 \\
\hline IV & 165 & 51,56 \\
\hline Sem informação & 18 & 5,62 \\
\hline \multicolumn{3}{|l|}{ Envolvimento linfonodal (N) } \\
\hline No & 148 & 46,40 \\
\hline N1 & 50 & 15,60 \\
\hline N2 & 72 & 22,40 \\
\hline N3 & 42 & 13,10 \\
\hline Sem informação & 8 & 2,50 \\
\hline \multicolumn{3}{|l|}{ Metástase à distância (M) } \\
\hline Mo & 269 & 84,10 \\
\hline M1 & 1 & 0,30 \\
\hline Sem informação & 50 & 15,60 \\
\hline \multicolumn{3}{|l|}{ Gradação histopatológica - OMS } \\
\hline Bem diferenciado & 31 & 9,69 \\
\hline Moderadamente diferenciado & 272 & 85 \\
\hline Pouco diferenciado & 12 & 3,75 \\
\hline Sem informação & 5 & 1,56 \\
\hline
\end{tabular}


Tabela 2 - Distribuição das variáveis em relação ao Estádio Clínico, Gradação Histopatológica e Óbito. As análises foram feitas pelo teste do qui-quadrado e teste exato de Fisher.

Table 2 - Distribution of variables according to Clinical Staging, Pathologic Grading and Death. Statistical analysis was performed through chi-square and Fisher's exact tests.

\begin{tabular}{|c|c|c|c|c|c|c|c|c|c|}
\hline \multirow[t]{3}{*}{ Característica } & \multicolumn{3}{|c|}{ Estádio Clínico } & \multicolumn{3}{|c|}{ Gradação OMS } & \multicolumn{3}{|c|}{ Óbito } \\
\hline & I e II & III e IV & & $B+M$ & $\mathbf{P}$ & & $\operatorname{sim}$ & não & \\
\hline & $\mathbf{n}$ & $\mathbf{n}$ & $\mathbf{p}$ & $\mathbf{n}$ & $\mathbf{n}$ & $\mathbf{p}$ & $\mathbf{n}$ & $\mathbf{n}$ & $\mathbf{p}$ \\
\hline \multicolumn{10}{|l|}{ Sexo } \\
\hline Feminino & 22 & 40 & & 60 & 3 & & 38 & 28 & \\
\hline Masculino & 59 & 181 & 0,107 & 242 & 9 & 0,713 & 144 & 110 & 1 \\
\hline \multicolumn{10}{|l|}{ Idade } \\
\hline$<60$ & 45 & 134 & & 181 & 7 & & 115 & 77 & \\
\hline$\geq 60$ & 35 & 76 & 0,28 & 110 & 4 & 1 & 61 & 55 & 0,235 \\
\hline \multicolumn{10}{|l|}{ Raça } \\
\hline Brancos & 47 & 93 & & & & & 74 & 75 & \\
\hline Pardos & 24 & 87 & & & $\mathbf{x}$ & & 78 & 41 & \\
\hline Negros & 8 & 40 & 0,025 & & & & 27 & 22 & 0,033 \\
\hline \multicolumn{10}{|l|}{ Tabagismo } \\
\hline Sim & 66 & 184 & & 247 & 11 & & 153 & 109 & \\
\hline Não & 15 & 37 & 0,732 & 55 & 1 & 0,7 & 29 & 29 & 0,246 \\
\hline \multicolumn{10}{|l|}{ Etilismo } \\
\hline$\underline{\operatorname{Sim}}$ & 48 & 150 & & 194 & 12 & & 120 & 89 & \\
\hline Não & 33 & 71 & 0,174 & 108 & 0 & 0,01 & 62 & 49 & 0,813 \\
\hline \multicolumn{10}{|l|}{ Língua } \\
\hline Sim & 46 & 113 & & 159 & 7 & & 96 & 73 & \\
\hline Não & 35 & 107 & 0,436 & 142 & 5 & 0,775 & 85 & 65 & 1 \\
\hline \multicolumn{10}{|l|}{ Assoalho } \\
\hline Sim & 29 & 105 & & 131 & 6 & & 80 & 59 & \\
\hline Não & 52 & 116 & 0,089 & 171 & 6 & 0,769 & 102 & 79 & 0,909 \\
\hline \multicolumn{10}{|c|}{ Região Retromolar } \\
\hline Sim & 10 & 46 & & 55 & 2 & & 33 & 25 & \\
\hline Não & 71 & 174 & 0,097 & 246 & 10 & 1 & 148 & 113 & 1 \\
\hline \multicolumn{10}{|c|}{ Gengiva Inferior } \\
\hline Sim & 9 & 52 & & 59 & 3 & & 29 & 35 & \\
\hline Não & 72 & 166 & 0,015 & 240 & 9 & 0,712 & 151 & 102 & 0,048 \\
\hline \multicolumn{10}{|c|}{ Gengiva Superior } \\
\hline Sim & 2 & 15 & & 19 & 1 & & 8 & 12 & \\
\hline Não & 79 & 203 & 0,171 & 280 & 11 & 0,556 & 172 & 125 & 0,161 \\
\hline \multicolumn{10}{|l|}{ Mucosa Jugal } \\
\hline Sim & 3 & 27 & & 30 & 0 & & 20 & 12 & \\
\hline$\underline{\text { Não }}$ & 78 & 191 & 0,029 & 269 & 12 & 0,614 & 160 & 125 & 0,574 \\
\hline \multicolumn{10}{|l|}{ Palato } \\
\hline Sim & 79 & 193 & & 32 & 1 & & 23 & 10 & \\
\hline Não & 2 & 25 & 0,013 & 267 & 11 & 1 & 157 & 127 & 0,138 \\
\hline
\end{tabular}


Tabela 2 - Distribuição das variáveis em relação ao Estádio Clínico, Gradação Histopatológica e Óbito. As análises foram feitas pelo teste do qui-quadrado e teste exato de Fisher. (continuação)

Table 2 - Distribution of variables according to Clinical Staging, Pathologic Grading and Death. Statistical analysis was performed through chi-square and Fisher's exact tests. (continuation)

\begin{tabular}{|c|c|c|c|c|c|c|c|c|c|}
\hline \multirow[t]{3}{*}{ Característica } & \multicolumn{3}{|c|}{ Estádio Clínico } & \multicolumn{3}{|c|}{ Gradação OMS } & \multicolumn{3}{|c|}{ Óbito } \\
\hline & Ie II & III e IV & & $B+M$ & $\mathbf{P}$ & & $\operatorname{sim}$ & não & \\
\hline & $\mathbf{n}$ & $\mathbf{n}$ & $\mathbf{p}$ & $\mathbf{n}$ & $\mathbf{n}$ & $\mathbf{p}$ & $\mathbf{n}$ & $\mathbf{n}$ & $\mathbf{p}$ \\
\hline \multicolumn{10}{|l|}{ Boca } \\
\hline Sim & 0 & 5 & & 5 & 0 & & 2 & 3 & \\
\hline Não & 81 & 214 & 0,329 & 295 & 12 & 1 & 179 & 134 & 0,655 \\
\hline \multicolumn{10}{|l|}{ Tratamento } \\
\hline Cirurgia & 17 & 14 & & & & & 9 & 23 & \\
\hline radioterapia & 15 & 113 & & & $x$ & & 91 & 44 & \\
\hline associação & 39 & 14 & $<0,0001$ & & & & 46 & 39 & $<0,0001$ \\
\hline \multicolumn{10}{|l|}{ Espera* (dias) } \\
\hline até 70 & 37 & 104 & & 143 & 4 & & 83 & 65 & \\
\hline$>70$ & 35 & 70 & 0,258 & 103 & 3 & 1 & 67 & 43 & 0,447 \\
\hline
\end{tabular}

palato duro $(0,013)$ - com grande parte dos tumores nessas localizações em estádios III ou IV - e com tratamento $(\mathrm{p}<0,0001)$, sendo a radioterapia exclusiva mais comumente indicada para tumores em estádio avançado. A gradação histopatológica, por sua vez, obteve associação apenas com o etilismo ( $\mathrm{p}=0,01)$, sendo mais comum a presença de tumores bem e moderadamente diferenciados nessa amostra. Já o óbito demonstrou associação com a raça ( $\mathrm{p}=0,033)$, com a localização em gengiva inferior ( $\mathrm{p}=0,048)$, $\mathrm{e}$ com o tratamento $(\mathrm{p}<0,0001)$ (Tabela 2$)$.

O pequeno número de casos pouco diferenciados da gradação histopatológica impossibilitou associação estatística entre a variável gradação da OMS e as variáveis raça e tratamento (Tabela 2 ).

\section{Análise de Sobrevida Global}

A média de tempo de acompanhamento foi de 29,5 meses. Em 13 casos não foi possível obter dados a respeito do acompanhamento do paciente e 3 pacientes tiveram acompanhamento inferior a 0,1 mês. O acompanhamento dos restantes variou de 0,1 a 82,1 meses. Ao final do período de estudo, 56,9\% dos pacientes evoluíram para óbito.

\section{Análise Univariada}

Dentre todas as variáveis analisadas, as que influenciaram significativamente a sobrevida, pela análise de Kaplan-Meier e pelo teste do log rank, foram o estádio, T e $\mathrm{N}$ (do TNM), tratamento e localização em mucosa jugal e palato.

\section{- Estádio:}

Quando comparadas as curvas de sobrevida dos pacientes diagnosticados em estádio inicial (I/II) e avançado (III/IV), a diferença de sobrevida entre esses dois grupos torna-se mais evidente a partir do terceiro ano de acompanhamento. A diferença entre as curvas, analisada pelo teste de $\log$ rank, foi significativa (log rank $=12,8$; g.l = 1 e valor $\mathrm{p}=0,0003$ - gráfico 1 ), comprovando que os pacientes diagnosticados em 


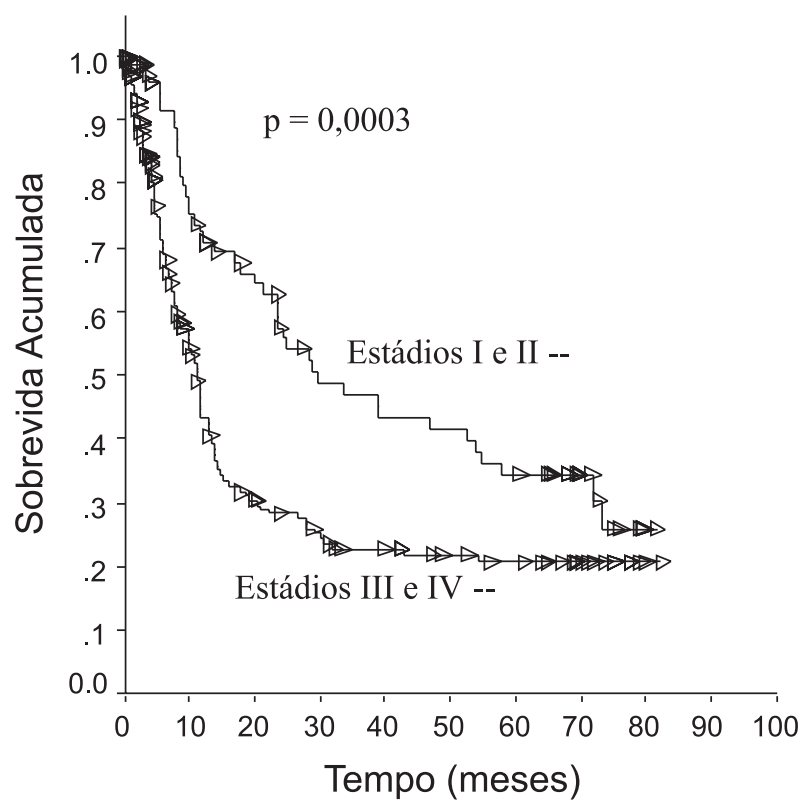

Gráfico 1 - Curvas obtidas pela análise de Kaplan-Meier para sobrevida global em relação ao estádio clínico.

Graph 1 - Kaplan-Meier curves for TNM clinical staging according to overall survival.

estádio avançado possuem pior sobrevida global (média de 25,4 meses, com erro padrão $=2,4)$ do que aqueles diagnosticados em estádio inicial (sobrevida média de 41,3 meses, erro padrão $=3,8$ ).

- Tamanho da lesão:

Quanto ao tamanho da lesão, os resultados refletem o esperado, pois as lesões de menor tamanho possuem melhor sobrevida $(\log$ rank $=28,12$, g.l $=3$, valor $\mathrm{p}<0,0001)$. Aos 60 meses, há uma inversão entre as curvas dos tumores $\mathrm{T} 1$ e T2 . A sobrevida média dos pacientes com tumores $\mathrm{T} 1, \mathrm{~T} 2$, T3 e T4 foi de 44,1 meses (erro padrão= 5,4), 37,4 meses (erro padrão $=3,7$ ), 27,3 meses (erro padrão $=4,1)$ e 18,6 meses (erro padrão $=$ $3,0)$, respectivamente.

- Acometimento de linfonodos:

Os pacientes que ao exame clínico apresentavam metástase em um único linfonodo (N1) obtiveram a melhor sobrevida (média de 36,1 meses, erro padrão $=5,5$ ), contrastando com os pacientes N3, que obtiveram a pior sobrevida global (média de 10,7 meses e erro padrão $=3,1$ ). A curva dos pacientes que não possuíam linfonodos acometidos (N0) apresentou comportamento diferenciado quando comparado aos outros níveis de acometimento . Entre o terceiro e quarto ano, a curva apresentou uma queda significativa, ultrapassando os valores de óbitos de pacientes N1, que apresentaram a melhor sobrevida média quando comparada aos pacientes classificados como N0 (sobrevida média de 34,9 meses e erro padrão $=2,0$ ) e N2 (sobrevida média de 25,0 meses e erro padrão = $4,6)$. O valor de log rank para esta análise foi 44, 0 , com g.l $=3$, com valor $\mathrm{p}<0,0001$.

- Tratamento:

Os pacientes tratados com cirurgia exclusiva obtiveram melhor sobrevida global quando comparados às outras formas de terapia (log rank 69, 31, com g.l = 2 com valor $\mathrm{p}<0,0001$ ) (Gráfico 2). A sobrevida média dos pacientes submetidos à cirurgia exclusiva foi de 59,4 meses (erro padrão = $6,1)$. Os submetidos à radioterapia exclusiva tiveram média de sobrevida de 15,6 meses (erro padrão $=1,9$ ), e quando houve associação de terapias, a sobrevida foi de 44,1 meses (erro padrão $=3,6$ ). 


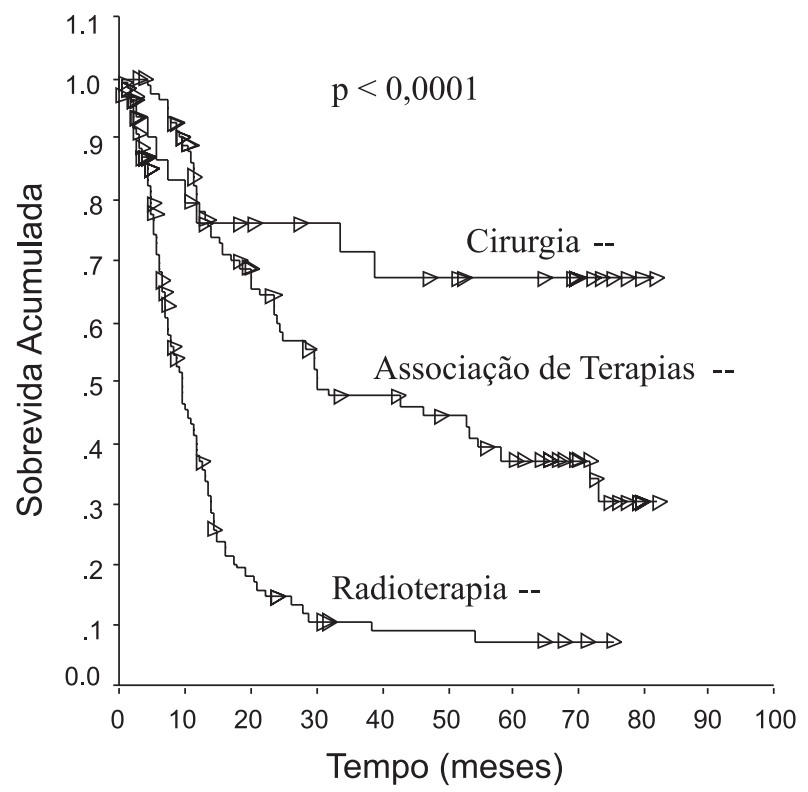

Gráfico 2 - Curvas obtidas pela análise de Kaplan-Meier para sobrevida global em relação ao tratamento.

Graph 2 - Kaplan-Meier curves for treatment modalities according to overall survival.

- Mucosa jugal e palato:

Os pacientes cujos tumores estavam localizados tanto em palato duro quanto em mucosa jugal apresentaram pior sobrevida global quando comparados aos pacientes que não possuíam tumores nessas localizações. As sobrevidas médias foram 16,2 meses (erro padrão $=4,3$ ) e 13,9 meses (erro padrão $=4,1$ ) para palato duro (log rank 7,64; g.l = $1 \mathrm{com}$ valor p 0,0057) e mucosa jugal (log rank 8,09; $\mathrm{gl}=1$ com valor $\mathrm{p} 0,0045)$, respectivamente.

As variáveis sexo, raça, tabagismo, etilismo, tempo de espera, gradação histopatológica, localização em língua, assoalho, região retromolar, gengiva inferior ou superior e áreas não especificadas da boca, não apresentaram valores estatisticamen- te significativos $(\mathrm{p}<0,05)$ pela análise de Kaplan-Meier utilizando o teste de log rank para comparação das curvas.

\section{Análise Multivariada}

Quando submetidas à análise multivariada, pelo modelo de regressão de Cox, verificou-se que, de todas as variáveis significativas pelo log rank, apenas tamanho ( $\mathrm{T}$ do TNM clínico), linfonodos ( $\mathrm{N}$ do TNM clínico) e localização em mucosa jugal foram consideradas como variáveis independentes relacionadas à sobrevida global nesse estudo, sendo que os pacientes com tumores T3 ou T4, com linfonodos acometidos e aqueles localizados em mucosa jugal apresentaram a pior sobrevida (Tabela 3).

Tabela 3 - Análise multivariada da sobrevida global pelo modelo de regressão de Cox.

Table 3 - Multiple regression analysis of overall survival by the Cox proportional hazards model.

\begin{tabular}{lccc}
\hline Covariáveis & Risco Relativo & $\begin{array}{c}\text { Intervalo de } \\
\text { Confiança de 95\% }\end{array}$ & $\mathrm{p}$ \\
\hline Tamanho (T) & 1,508 & $1,188-1,913$ & 0,001 \\
Linfonodos (N) & 1,27 & $1,054-1,530$ & 0,012 \\
Mucosa Jugal & 1,948 & $1,107-3,431$ & 0,021 \\
\hline
\end{tabular}




\section{Discussão}

O perfil epidemiológico da amostra estudada coincide com a literatura ${ }^{2,6,9}$. O sexo masculino é o mais afetado, provavelmente devido a maior exposição aos fatores de risco. A associação de álcool e tabaco, menos comum entre mulheres, nitidamente influencia a presença da doença nessa população. Além disso, quando ocorre tal associação, o uso do tabaco ocorre de forma mais branda. Este trabalho demonstra consenso com a literatura ${ }^{2,6,9}$ em relação à faixa etária mais comum entre os pacientes acometidos, em torno da sexta década de vida. A língua, também em diversos estudos, surge como o sítio anatômico mais acometido, seguida do assoalho de boca ${ }^{9,10}$.

O diagnóstico em estádios avançados ocorreu, nesse estudo, em $68,9 \%$ dos casos, refletindo a demora pela procura de um serviço especializado ${ }^{11-13}$ ou a demora para se chegar a um diagnóstico definitivo. $\mathrm{O}$ prognóstico do CCE de boca melhora se for identificado em fases iniciais. Entretanto, há pouca ou nenhuma sintomatologia nessa fase, podendo apresentar aparência inofensiva, o que muitas vezes leva ao atraso em seu diagnóstico e tratamento ${ }^{5}$. Além disso, esses fatores podem fazer com que a lesão não seja valorizada pelo próprio indivíduo nem pelos profissionais de saúde ${ }^{14}$.

A modalidade terapêutica a ser aplicada depende do sítio e do estádio da doença, além do estado de saúde geral do paciente. Dessa forma, quanto mais precoce o diagnóstico, menos agressivo será o tratamento, proporcionando melhor estética e função das estruturas envolvidas ${ }^{15,16}$. Como a maior parte dos pacientes foi diagnosticada em estádios avançados, foi comum a indicação da radioterapia exclusiva (53,6\% dos casos) ou da associação de diferentes modalidades terapêuticas (33,7\% dos casos). A associação da radioterapia com pior sobrevida global pela análise de Kaplan-Meier não foi inesperada, tampouco com estádio avançado e maior número de óbitos pelo teste do qui-quadrado, pois geralmente são submetidos à radioterapia exclusiva os pacientes cujos tumores estão em localizações que não permitem ressecção cirúrgica ou em pacientes tão debilitados que não podem ser submetidos à cirurgia, ou ainda tumores em estádios avançados ${ }^{9,10}$.

Os pacientes com tumores muito avançados são submetidos a cuidados paliativos, significando que não serão adotadas as formas terapêuticas convencionais: radioterapia, cirurgia ou quimioterapia. Esses pacientes são encaminhados a uma unidade especial do Instituto Nacional do Câncer, onde recebem tratamento diferenciado, com possível melhora da qualidade de vida. Na análise da variável tratamento, tais pacientes não foram incluídos, assim como aqueles que se recusaram a receber tratamento ou o abandonaram após o diagnóstico, e ainda aqueles que faleceram antes de iniciar qualquer terapêutica.

O tamanho da lesão e a presença de linfonodos já foram considerados ineficazes como fatores prognósticos para pacientes com doença em estádio inicial ${ }^{17}$. Entretanto, quando o grupo avaliado possui tanto pacientes em estádio inicial quanto em estádio avançado, a presença de linfonodos acometidos está fortemente associada a uma redução da sobrevida para aproximadamente $50 \%{ }^{12,18}$, e por isso torna-se um fator prognóstico importante. O presente estudo analisou todos os estádios do CCE de boca, submetidos a diferentes modalidades terapêuticas, e encontrou que o tamanho e a presença de linfonodos ao diagnóstico (variáveis T e $\mathrm{N}$ do TNM clínico) foram as variáveis mais significativas para predizer sobrevida, tanto pela análise de KaplanMeier, utilizando o teste de log rank para comparação das curvas, quanto pelo modelo de Cox. Isso indica que o $\mathrm{T}$ e o $\mathrm{N}$ podem ser considerados fatores prognósticos independentes para predizer sobrevida global nesse grupo de pacientes, e que os pacientes sem linfonodos acometidos ao diagnóstico e com tumores $\mathrm{T} 1$ ou $\mathrm{T} 2$ possuem maior sobrevida.

$\mathrm{Na}$ análise multivariada utilizando o modelo de Cox, outro fator que se revelou significativo para predizer pior sobrevida 
global foi a localização em mucosa jugal. Diferentes estudos ${ }^{19,20}$ demonstraram que os tumores em mucosa jugal apresentam características de agressividade, com altos índices de recorrência loco-regional. Sieczka et al. afirmaram que mesmo os tumores em estágios iniciais localizados em mucosa jugal se beneficiariam da terapia adjuvante após a cirurgia ${ }^{20}$. Contudo, foi utilizado um número limitado de casos $(\mathrm{n}=27)^{20}$. No presente estudo, houve associação dos tumores de mucosa jugal com estádio clínico avançado ( $\mathrm{p}=0,03$ - Tabela 2). Estudos utilizando amostras maiores de tumores localizados em mucosa jugal poderão ajudar a esclarecer essa questão.

A sobrevida de 5 anos em pacientes submetidos à cirurgia é de aproximadamente $50 \%{ }^{21}$, chegando a atingir $83,3 \%{ }^{22}$ quando a sobrevida específica do câncer é analisada. Contudo, em estudos que incluem pacientes que foram tratados com diferentes modalidades terapêuticas, a sobrevida global em 5 anos diminui bastante, chegando a atingir $24 \%{ }^{23}$, valor semelhante ao encontrado na presente amostra. Foi observado (Gráfico 4) que a curva de pacientes que não possuíam linfonodos acometidos (N0) entre o terceiro e quarto ano de acompanhamento apresentou uma queda significativa, ultrapassando os valores de óbitos de pacientes N1. Os pacientes com pescoço negativo (N0) diagnosticado por palpação, e até mesmo por ressonância magnética, podem apresentar metástases ocultas ${ }^{24}$, que vêm a se manifestar sob a forma de recorrência regional e/ou óbito nesse período de acompanhamento. Porém, tais metástases deixam de ser diagnosticadas, pois os pacientes em estádio I, no período avaliado pelo presente estudo, não possuíam indicação de realizar esvaziamento cervical ${ }^{25}$, sendo esse procedimento indicado nos pacientes estadiados clinicamente como N1. Entretanto, estudos recentes ${ }^{26}$ demonstraram que pacientes com tumores de língua e assoalho nos estádio I e II submetidos a esvaziamento cervical supraomoioídeo têm a incidência de recorrência em linfonodos diminuída, o que vem tornando o esvaziamento cervi- cal eletivo uma indicação nesse grupo de pacientes.

Da forma como a $\mathrm{OMS}^{3,27}$ recomenda a aplicação da gradação histopatológica do CCE de boca, dividida em 3 graus - bem, moderadamente e pouco diferenciado apesar de ser frequentemente avaliada em estudos a respeito de fatores prognósticos, apenas em alguns estudos ${ }^{28-31}$ encontra-se relação com a sobrevida. Tampouco no presente estudo foi encontrada associação entre a gradação histopatológica da OMS e a sobrevida. A própria OMS reconhece as limitações em relação ao valor prognóstico da gradação indicada, destacando que a maioria dos tumores acaba sendo classificado como moderadamente diferenciado e sugere que outros fatores sejam levados em consideração, como o padrão de invasão e a condição dos linfonodos ${ }^{27}$. Além disso, devido ao pequeno número de tumores bem e pouco diferenciados, a análise do qui-quadrado não foi possível para algumas variáveis, mesmo agrupando os bem $\mathrm{e}$ moderadamente diferenciados.

A melhor forma de expressar o prognóstico desta neoplasia é analisar a taxa de mortalidade, estabelecendo índice de gravidade, tanto do ponto de vista clínico como de saúde pública. Contudo as comorbidades, frequentes nesse tipo de população devido à faixa etária prevalente e ao alto índice de tabagismo e etilismo, influenciam a decisão a respeito do tratamento e o desfecho final diretamente, diminuindo ainda mais os índices de sobrevida global desses pacientes ${ }^{32}$. Portanto, não basta tratar o câncer, mas melhorar o acesso aos cuidados de saúde para a população e aumentar a conscientização a respeito dos fatores de risco para essa doença e enfermidades relacionadas.

\section{Conclusões}

As taxas de sobrevida permanecem baixas na população estudada e o perfil epidemiológico dos pacientes condiz com o já descrito na literatura. A ausência de linfonodos acometidos ao diagnóstico e tumores de pequeno tamanho indicam me- 
lhor sobrevida, enquanto a localização em mucosa jugal indica pior sobrevida global. Isso justifica o estudo de fatores diagnósticos e prognósticos para o CCE de boca, além de campanhas e projetos que incentivem e promovam o diagnóstico precoce dessa neoplasia entre os profissionais da saúde.
Também deve haver a conscientização da população a respeito dos fatores de risco e da importância da realização de exames periódicos de inspeção da cavidade bucal.

\section{Conflito de interesses:}

Não há conflito de interesses a declarar.

\section{Referências}

1. INCA. Estimativa 2008 - Incidência de câncer no Brasil; 2008. Disponível em http://www.inca.gov.br/ estimativa/2008. (FALTA DADO DE ACESSO)

2. Costa JC, Nunes AAF, Arruda, MLS. Correlação entre a classificação TNM, gradação histológica e localização anatômica em carcinoma epidermóide oral. Pesqui Odontol Bras 2002; 16(3): 216-20.

3. Pindborg JR, PA; Smith, CJ. Histological typing of cancer and precancer of the oral mucosa. WHO International Classification of Tumors. 2 ed. Berlin: Springer Verlag; 1997.

4. Lockhart PB, Norris CM, Jr., Pulliam C. Dental factors in the genesis of squamous cell carcinoma of the oral cavity. Oral Oncol 1998 Mar; 34(2): 133-9.

5. Kowalski LP, Carvalho AL, Martins Priante AV, Magrin J. Predictive factors for distant metastasis from oral and oropharyngeal squamous cell carcinoma. Oral Oncol 2005; 41(5): 534-41.

6. La Vecchia C, Tavani A, Franceschi S, Levi F, Corrao G, Negri E. Epidemiology and prevention of oral cancer. Oral Oncol 1997; 33(5): 302-12.

7. Bettendorf O, Piffko J, Bankfalvi A. Prognostic and predictive factors in oral squamous cell cancer: important tools for planning individual therapy? Oral Oncol 2004; 40(2): 110-9.

8. Patel SG, Shah JP. TNM staging of cancers of the head and neck: striving for uniformity among diversity. $C A$ Cancer J Clin 2005; 55(4): 242-58; quiz 61-2, 64.

9. Leite IC, Koifman S. Survival analysis in a sample of oral cancer patients at a reference hospital in Rio de Janeiro, Brazil. Oral Oncol 1998; 34(5): 347-52.

10. Liao CT, Kang CJ, Chang JT, Wang HM, Ng SH, Hsueh C, et al. Survival of second and multiple primary tumors in patients with oral cavity squamous cell carcinoma in the betel quid chewing area. Oral Oncol 2006; (COMPLETAR REF. C/ No. REVISTA E DE PÁGS.)

11. Massano J, Regateiro FS, Januario G, Ferreira A. Oral squamous cell carcinoma: review of prognostic and predictive factors. Oral Surg Oral Med Oral Pathol Oral Radiol Endod 2006; 102(1): 67-76.
12. Preuss SF, Dinh V, Klussmann JP, Semrau R, Mueller RP, Guntinas-Lichius O. Outcome of multimodal treatment for oropharyngeal carcinoma: a single institution experience. Oral Oncol 2007; 43(4): 402-7.

13. Scott SE, Grunfeld EA, McGurk M. The idiosyncratic relationship between diagnostic delay and stage of oral squamous cell carcinoma. Oral Oncol 2005; 41(4): 396403.

14. Mashberg A, Samit A. Early diagnosis of asymptomatic oral and oropharyngeal squamous cancers. CA Cancer J Clin 1995; 45(6): 328-51.

15. Canto MT, Devesa SS. Oral cavity and pharynx cancer incidence rates in the United States, 1975-1998. Oral Oncol 2002; 38(6): 610-7.

16. Al-Rajhi N, Khafaga Y, El-Husseiny J, Saleem M, Mourad W, Al-Otieschan A, et al. Early stage carcinoma of oral tongue: prognostic factors for local control and survival. Oral Oncol 2000; 36(6): 508-14.

17. Veneroni S, Silvestrini R, Costa A, Salvatori P, Faranda A, Molinari R. Biological indicators of survival in patients treated by surgery for squamous cell carcinoma of the oral cavity and oropharynx. Oral Oncol 1997; 33(6): 40813.

18. Hashibe M, Ritz B, Le AD, Li G, Sankaranarayanan R, Zhang ZF. Radiotherapy for oral cancer as a risk factor for second primary cancers. Cancer Lett 2005; 220(2): 185-95.

19. Jing J, Li L, He W, Sun G. Prognostic predictors of squamous cell carcinoma of the buccal mucosa with negative surgical margins. J Oral Maxillofac Surg 2006; 64(6): 896-901.

20. Sieczka E, Datta R, Singh A, Loree T, Rigual N, Orner J, et al. Cancer of the buccal mucosa: are margins and T-stage accurate predictors of local control? Am J Otolaryngol 2001; 22(6): 395-9.

21. Kademani D, Bell RB, Bagheri S, Holmgren E, Dierks E, Potter B, et al. Prognostic factors in intraoral squamous cell carcinoma: the influence of histologic grade. J Oral Maxillofac Surg 2005; 63(11): 1599-605. 
22. Chandu A, Adams G, Smith AC. Factors affecting survival in patients with oral cancer: an Australian perspective. Int J Oral Maxillofac Surg 2005; 34(5): 514-20.

23. Oliveira LRd, Ribeiro-Silva A, Zucoloto S. Perfil da incidência e da sobrevida de pacientes com carcinoma epidermóide oral em uma população brasileira. J Bras Patol Med Lab 2006; 42(5): 385-92.

24. Woolgar JA. Pathology of the N0 neck. Br J Oral Maxillofac Surg 1999; 37(3): 205-9.

25. INCA/MS. Carcinoma Epidermóide da Cabeça e Pescoço - Condutas do INCA/MS. Rev Bras Canc 2001; 47(4): 36176.

26. Dias FL, Lima RA, Kligerman J, Farias TP, Soares JR, Manfro G, et al. Relevance of skip metastases for squamous cell carcinoma of the oral tongue and the floor of the mouth. Otolaryngol Head Neck Surg 2006; 134(3): 460-5.

27. Barnes L, Eveson J.W., Reichert P., Sidransky D. World Health Organization Classification of Tumours. Pathology and Genetics of Head and Neck Tumours. Lyon: IARC Press; 2005.
28. Garzino-Demo P, Dell'Acqua A, Dalmasso P, Fasolis M, La Terra Maggiore GM, Ramieri G, et al. Clinicopathological parameters and outcome of 245 patients operated for oral squamous cell carcinoma. $J$ Craniomaxillofac Surg 2006; 34(6): 344-50.

29. Homma A, Furuta Y, Oridate N, Nakano Y, Kohashi G, Yagi K, et al. Prognostic significance of clinical parameters and biological markers in patients with squamous cell carcinoma of the head and neck treated with concurrent chemoradiotherapy. Clin Cancer Res 1999; 5(4): 801-6.

30. Lo Muzio L, Farina A, Rubini C, Pezzetti F, Stabellini G, Laino $\mathrm{G}$, et al. Survivin as prognostic factor in squamous cell carcinoma of the oral cavity. Cancer Lett 2005; 225(1): 27-33.

31. Rahima B, Shingaki S, Nagata M, Saito C. Prognostic significance of perineural invasion in oral and oropharyngeal carcinoma. Oral Surg Oral Med Oral Pathol Oral Radiol Endod 2004; 97(4): 423-31.

32. Hall SF, Groome PA, Rothwell D. The impact of comorbidity on the survival of patients with squamous cell carcinoma of the head and neck. Head Neck 2000; 22(4): 317-22. 